\title{
Exploring the Depths: Matching Light Microscopy Techniques to Applications
}

\author{
Samuel Bonfig* and Alec De Grand
}

Olympus Scientific Solutions Americas, 48 Woerd Ave., Waltham, MA 02453

*samuel.bonfig@olympus.com

Abstract: Modern light microscopy techniques have specific advantages for the analysis of tissues and organisms that were unknown just a few decades ago. One important capability is the capture of images from greater depths within a specimen than ever before. This brief review summarizes the capabilities of fluorescence microscopy, total internal reflection fluorescence (TIRF) microscopy, confocal microscopy, multiphoton microscopy, and super-resolution microscopy. In addition, clearing agents and special objective lenses are described that allow image capture from a depth of several millimeters in cleared tissue.

\section{Introduction}

Few researchers have the opportunity to build their entire laboratory from scratch; most work in laboratories where much of the equipment is already in place and only supplemental grants for select instrumentation are available. The microscopes in most life science research laboratories have become imaging workhorses, sometimes remaining in use for decades. But how does a scientist ascertain whether what is already in the laboratory is sufficient to meet the needs of the science he or she will be performing? Selecting an optimal microscopy technique depends on many things, such as the desired depth of imaging, resolution and magnification needs, ease of training, hardware expandability, and software capabilities.

All imaging techniques seek to maximize signal and decrease noise, and each experiment has its own set of challenges. For live specimens, the faster an organism moves or the deeper a region of interest is below the tissue surface, the greater the imaging challenge can be. Light scatter, absorption, background signal, and differences in refractive index present a variety of hurdles, but every imaging technique can be optimized with a system designed to meet particular needs. The following is an overview of various microscope imaging techniques employed by the scientific community, with some of the benefits, drawbacks, and key applications of each technique. Of particular interest is the imaging depth associated with each method.

\section{Fluorescence Microscopy}

The go-to technique for biological imaging in many research laboratories is widefield epifluorescence microscopy. Researchers use fluorescence imaging to capture the immunostaining of membranes, organelles, proteins, DNA, and many other biological structures, enabling the imaging of life processes as they occur. In fluorescence microscopy, specimens are illuminated with specific wavelengths of light. This light is absorbed by fluorophores, also called fluorescent probes, that have been attached to specimen features of interest; this absorption triggers the fluorophores to emit specific, longer wavelengths of light. It is this emitted fluorescent light that is detected by the microscope. Fluorescence images are routinely captured in grayscale using a photomultiplier tube (PMT); this light detector optimizes sensitivity for dimly fluorescing specimens.

Widefield fluorescence microscopes offer bright, sharp images at a relatively low price point (Figure 1). Fluorescence microscopy has the ability to detect low concentrations of specific fluorophores over time with single-molecule resolution. A tremendous variety of fluorophores is available, allowing users to incorporate fluorescence at different wavelengths to create striking multicolor images.

However, widefield fluorescence has its drawbacks. Repeated exposure of the specimen to light often leads to photobleaching, which reduces fluorescence emission and degrades image contrast quickly. It also can be difficult to generate enough fluorescence to gather sufficient data. Biological specimens typically emit fluorescence signals three to six orders of magnitude less than that of the illumination, making molecule detection challenging, particularly at greater depths [1]. Increasing the amount of light in order to get more signal out of the specimen also can be dangerous; the shorter-wavelength light used in fluorescent imaging can be phototoxic to living cells. Finally, the inability of widefield epifluorescence to reduce background noise outside the focal plane makes it generally unsuitable at depths greater than $700 \mathrm{~nm}$.

\section{TIRF Microscopy}

One specialized form of fluorescence is designed for imaging just below the surface of a cover slip where the cell membrane

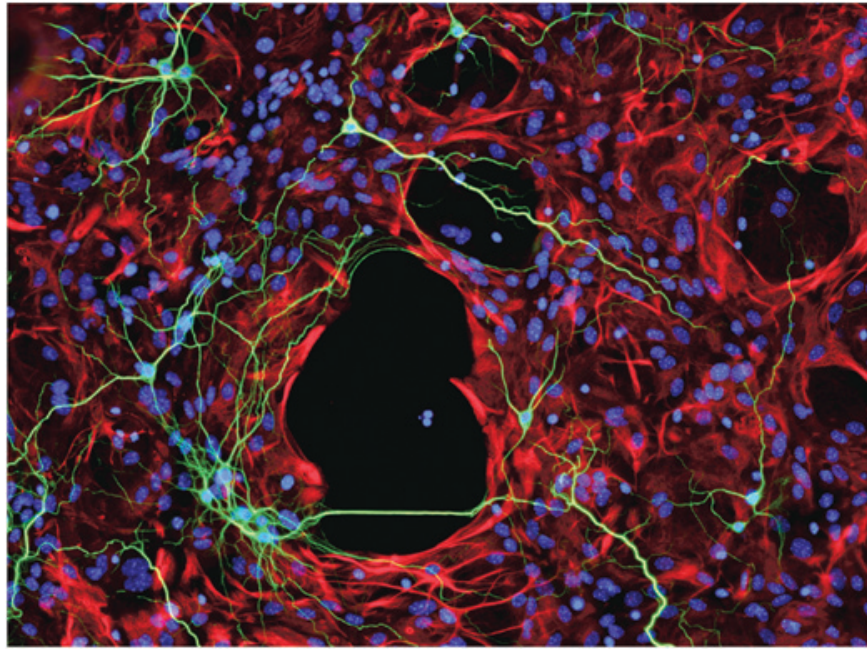

Figure 1: Mouse neurons and astrocytes captured using immunofluorescence light microscopy. Neurons (green) were stained with anti-MAP2 and Alexa Fluor 488, astrocytes (red) were stained with anti-GFAP and Alexa Fluor 568, and nuclei (blue) were stained with DAPI. Image width $=4340 \mu \mathrm{m}$. Courtesy of of James Striebel and Melissa Pathmajeyan, National Institutes of Health, Corvallis, MT. Honorable Mention, 2007 Olympus BioScapes Digital Imaging Competition. 


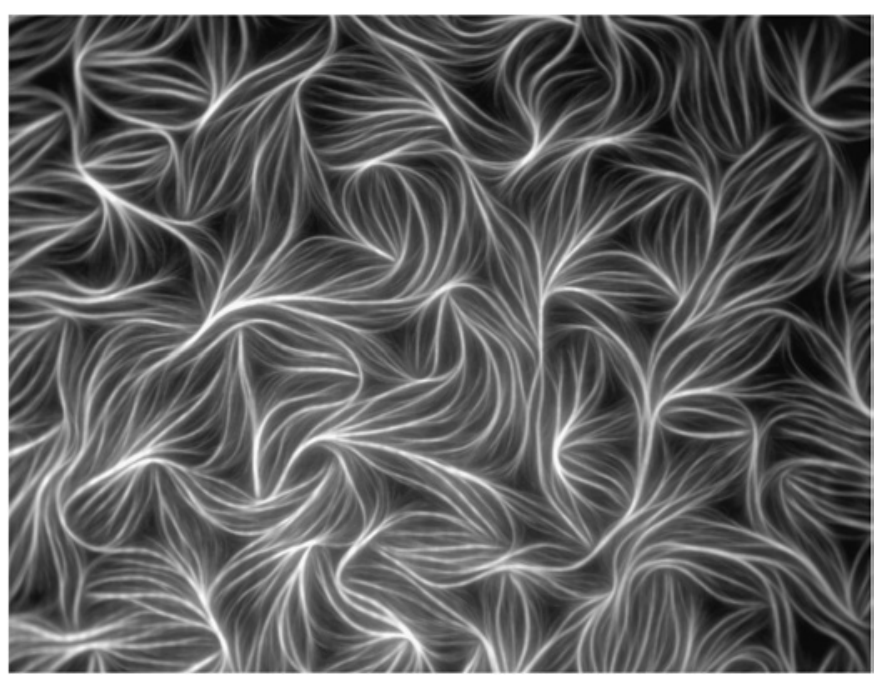

Figure 2: TIRF image of fluorescently labeled actin from chicken muscle. Dennis Breitsprecher, Hannover Medical School, Institute of Biophysical Chemistry, Hannover, Germany. Honorable Mention, 2010 Olympus BioScapes Digital Imaging Competition.

adheres. Total internal reflection fluorescence (TIRF) imaging (Figure 2) requires a research-grade fluorescence microscope, a specialized prism, and a laser. At a specific angle, the beam of laser light passing through the objective is totally reflected at the glass/specimen interface, rather than passing through; this is called the critical angle. This angle is very shallow relative to the interface and requires the objective lens to have a very high numerical aperture (greater than 1.45). Reflection at the critical angle produces an evanescent wave, which propagates into the aqueous medium. This evanescent wave has an identical wavelength to that of the incident light. The critical angle can vary depending on the materials involved, but the strength of this evanescent wave decreases exponentially as it extends through the second medium [2].

TIRF microscopy is a powerful way of elucidating processes occurring at the cell surface, such as membrane adhesion and movement, cell interactions, protein secretion, and adsorption. The shallow penetration depth of the evanescent wave allows only those fluorophores near the surface of a specimen to become excited, creating a thin optical section that can readily be imaged [3]. Although it is only applicable at shallow depths (to $200 \mathrm{~nm}$ ), TIRF is an excellent technique for obtaining high-contrast images with excellent signal-to-noise ratios because light penetration outside the evanescent field is minimal.

\section{Confocal Microscopy}

Thick, living tissue can sometimes scatter so much light that very little specimen-emitted fluorescence reaches the detector, and the challenges of photobleaching and phototoxicity remain daunting. As specimens become more three-dimensional and regions of interest demand extreme depths, traditional fluorescence techniques reach their limit of effectiveness.

Confocal microscopy removes out-of-focus light originating from outside the focal plane, permitting thin optical sectioning

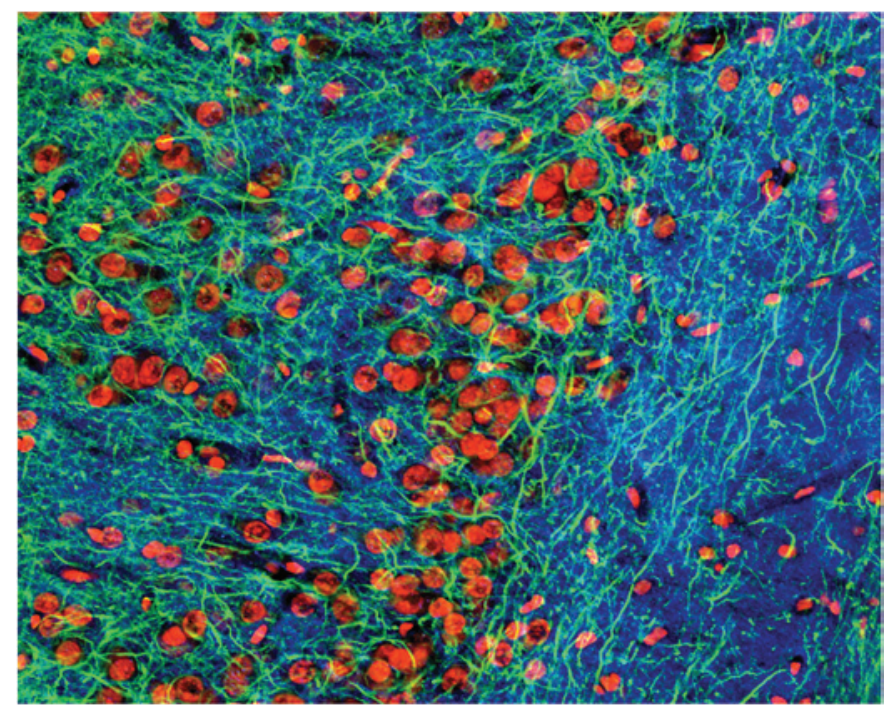

Figure 3: Confocal image of neurofilaments in coronal rat brain section Image courtesy of the late Michael W. Davidson, Florida State University, Tallahassee, FL.

(Figure 3). This makes confocal imaging ideal for live-cell fluorescence imaging at a multitude of depths. Confocal systems are widely used in life science laboratories because they allow scientists to study biological processes in real time. Also, by imaging stacks of individual sections created from optical slices, researchers can create three-dimensional images with good resolution in each dimension.

But even confocal systems have their limits; excitation light generates fluorescence throughout the entire depth of the sample, including all the areas above and below the region of interest, often photobleaching and damaging the entire volume of a specimen [4]. Also, collecting the emitted signal can be complex. Emitted photons from the focal plane must travel from the sample through the microscope's optical system and then pass through a small slit or pinhole, called the confocal aperture, before arriving at the detector. Any signal that comes from outside the focal plane is removed by the confocal aperture. Scattering or deflection of the fluorescence as it passes through the sample can easily result in the light being removed by the confocal aperture. Photons traveling from deep within the specimen experience higher levels of scattering and rejection compared to shallower objects, making deep imaging a challenge.

\section{Multiphoton Microscopy}

Where confocal imaging falls short, multiphoton systems excel. Multiphoton systems allow imaging at much deeper levels than their confocal relatives, typically up to about $700-1,000 \mathrm{~nm}$ below the surface. Typically longer wavelengths of light are used in multiphoton systems because these wavelengths scatter less when moving through tissue and are not as harmful to living cells.

In multiphoton imaging, multiple long-wavelength, lowenergy excitation photons are absorbed simultaneously by a single fluorophore at a specific point location in $x, y$ and $z$ [5]. When this happens, the photons combine their energies as 


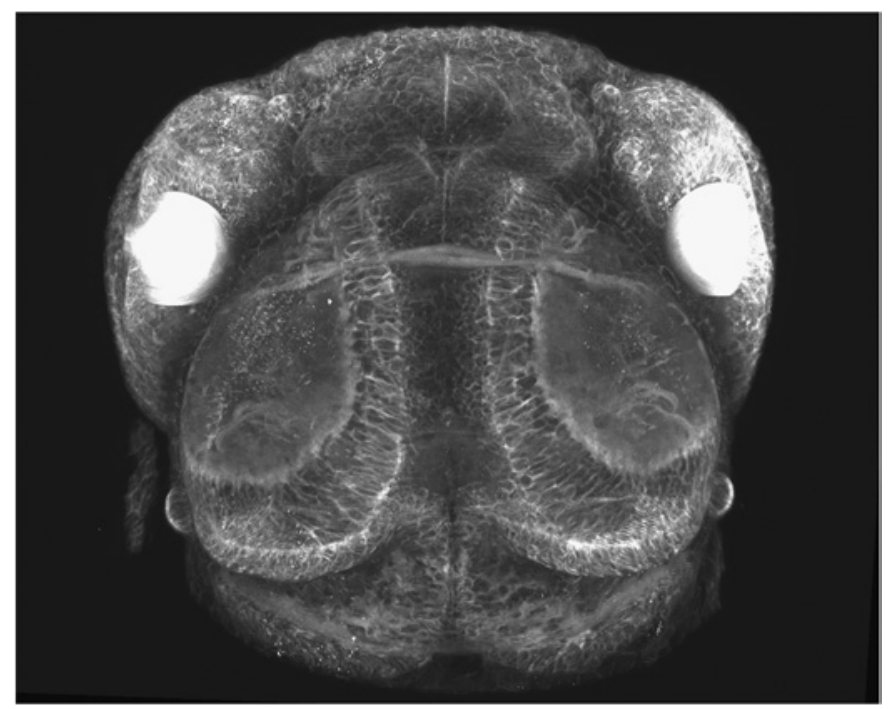

Figure 4: Maximum intensity projection of live zebrafish transgenically expressing yellow fluorescent protein (YFP), showing the optic nerves. Zebrafish head is $350 \mu \mathrm{m}$ wide. Image acquired in grayscale with $4 \mathrm{~mm} 25 \times$ objective. Image courtesy of Phillip Williams and Rachel Wong, University of Washington, Seattle, WA.

if they were a single excitation photon of high energy (short wavelength). This combined-energy light excites the fluorophore and stimulates fluorescence, just as if the fluorophore had been stimulated by illumination of higher energy. Because the long-wavelength illumination photons coincide only at the location of interest, multiphoton fluorescence excitation occurs only at the plane of focus at a single point. This differs from confocal microscopy, where fluorescence is generated throughout the entire volume of the specimen. As a result, there is less phototoxicity and photobleaching, and the specimen can be imaged for a longer period of time. Multiphoton microscopy allows the imaging of brain slices, eye tissue, developing embryos, and other living tissue that is exceptionally thick or dense (Figure 4).

Cell biologists, neuroscientists, physiologists, and other researchers who wish to study dynamic processes over time in living cells and tissues use multiphoton systems to keep specimens alive for as long as possible while imaging life processes. Often, such studies last days, weeks, or even longer. Because of this, in-vivo imaging with multiphoton excitation is becoming one of the technique's main applications.

\section{Clearing Technologies}

Until recently, it was impossible to image deeper than one millimeter into biological tissue using a light microscope, but the clearing methodologies developed over the past five years have allowed researchers to image up to $8,000 \mathrm{~nm}(8 \mathrm{~mm})$ deep using fixed specimens. These new systems use multiphoton microscopes, ultra-long-working-distance optics, and proprietary clearing reagents to facilitate the imaging of contiguous fixed tissue deep within dense organs.

In August 2011, a team at the RIKEN Brain Science Institute in Japan led by Dr. Atsushi Miyawaki published data in Nature Neuroscience [6] showing that they had created a clearing agent called SCALE, which, when used with a newly developed

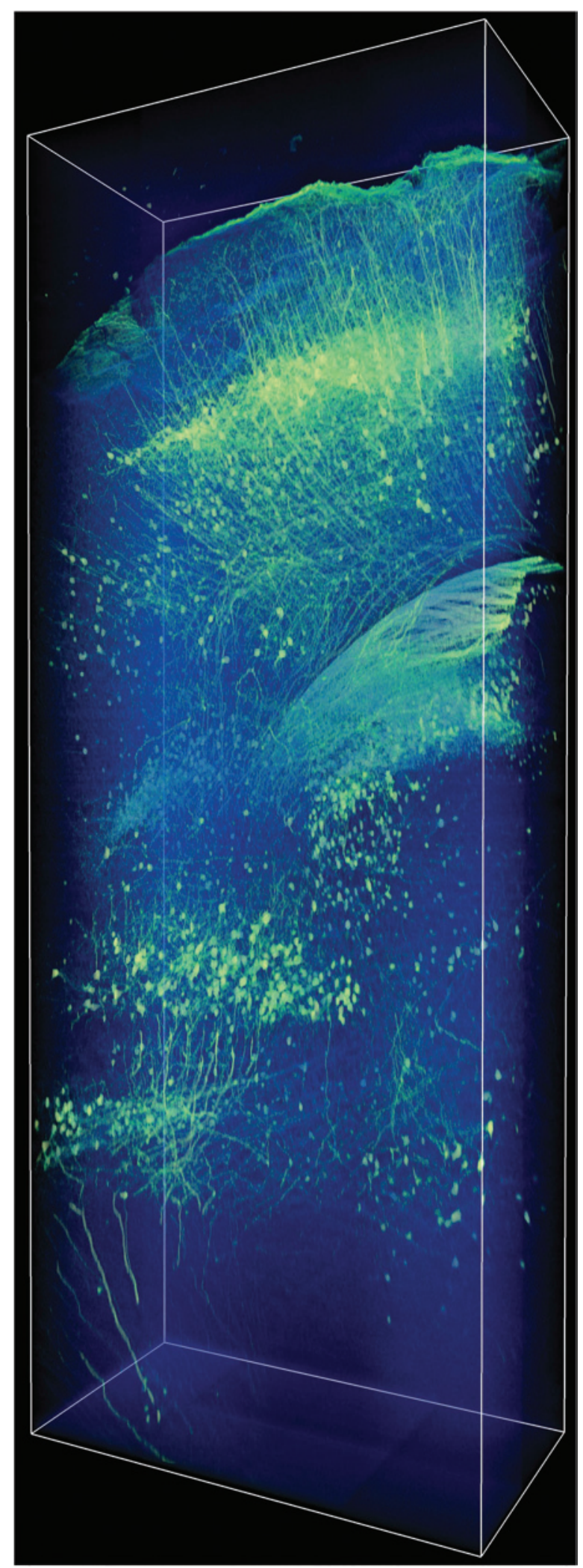

Figure 5: Fast high-resolution imaging of clarified brain using CLARITYoptimized light-sheet microscopy (COLM). The $1 \mathrm{~mm} \times 2 \mathrm{~mm} \times 5 \mathrm{~mm}$ volume was acquired from an intact clarified Thy1-EYFP mouse brain using COLM with $25 \times$ magnification. Camera exposure time of $20 \mathrm{~ms}$ was used; RI liquid 1.454 was used as the immersion medium. Image courtesy of Raju Tomer, Stanford University School of Medicine, Stanford, CA. 
$25 \times$ multiphoton Olympus SCALEVIEW objective, allowed the researchers to observe contiguous brain tissue up to $8 \mathrm{~mm}$ deep, several times deeper than ever before. Lens focus through even the deepest tissue was assured with specialized correction collars on the objectives [7].

The announcement of SCALE was followed in 2013 by CLARITY, which came out of Karl Deisseroth's laboratory at Stanford University [8]. CLARITY, another promising clearing technique, involves creating a stable tissue-hydrogel hybrid out of thick biological tissue without changing the tissue's structure [9]. CLARITY has the potential to help researchers extract vital neurobiological data from intact tissue deep within specimens (Figure 5).

Clearing techniques such as SCALE and CLARITY are significant because they allow researchers to visualize neurological connections through the dense, light-scattering tissue of the brain. Because they do not require specimen slicing, these clearing reagants have improved the ability to map neural pathways continuously. Although these deep-imaging techniques have great potential, they are currently limited to fixed-tissue applications.

\section{Super-Resolution Microscopy}

Researchers are always seeking to enhance resolution at any depth within a specimen. Super-resolution light microscopy includes a wide spectrum of imaging software, hardware, and methodologies that can improve the lateral $(x, y)$ resolution of a microscope at various depths. Such resolution can even extend beyond the theoretical diffraction limit of a light optical system [10]. Many super-resolution techniques employ timeresolved localization of photoswitchable fluorophores and sequential activation to build ultra-high-resolution images from acquired data.

While super resolution can be achieved by selectively deactivating fluorophores, as in stimulated emission depletion (STED) microscopy [11], other techniques provide benefits with respect to axial resolution (depth). Structured illumination microscopy (SIM) is an example. First demonstrated by a group of Janelia Farm researchers in 2008 [12], SIM scanners have been shown to achieve $z$-axis resolution to about $300 \mathrm{~nm}$ [13].

Super-resolution imaging has enormous potential for helping researchers image phenomena they could not see before. These methodologies present challenges as well. Most super-resolution techniques require expensive hardware such as specialized photoswitching probes and specific laser systems designed for localization [14]. They also involve rigorous sample preparation, making the methodologies difficult to implement in some laboratories. In addition, super resolution depends on the use of complex mathematical models, making its computational, storage, and upkeep requirements challenging. Several companies now offer affordable software-based options that provide some of the benefits of hardware-based super resolution.

\section{Conclusion}

From capturing processes at the surface of a membrane to resolving phenomena at unprecedented depths, light microscopy has evolved significantly over the past decade. Only after identifying the parameters of importance to the phenomena under study and understanding the pros and cons of each prospective microscopy technique can the best imaging methodology and equipment be matched to a given application.

\section{References}

[1] D Murphy and MW Davidson, Fundamentals of Light Microscopy and Electronic Imaging, 2nd ed., Wiley-Blackwell, Hoboken, 2013.

[2] NL Thompson et al., Biophys J 33 (1981) 435-54.

[3] E Betzig et al., Science 313 (2006) 1642-45.

[4] J Pawley, ed., Handbook of Biological Confocal Microscopy, 3rd ed., Springer, New York, 2006.

[5] W Denk et al., Science 248 (1990) 73-76.

[6] H Hama et al., Nat Neurosci 14 (2011) 1481-88.

[7] B Brinkman, Microscopy Today 21 (2013) 10-13.

[8] K Chung and K Deisseroth, Nat Methods 10 (2013) $508-13$.

[9] R Tomer et al., Nat Protoc 9 (2014) 1682-97.

[10] JA Conchello and JW Lichtman, Nat Methods 2 (2005) 920-31.

[11] SW Hell and J Wichmann, Opt Lett 19 (1994) 780-82.

[12] L Shao et al., Nat Methods 8 (2011) 1044-46.

[13] MGL Gustafsson, P Natl Acad Sci 102 (2005) 13081-86.

[14] MJ Rust et al., Nat Methods 3 (2006) 793-96.

Expand your Knowledge of Microscopy with MSA Membership!

Whether your primary focus is in the biological or the physical sciences, MSA takes your knowledge to the next level!

Members Receive:

-A personal subscription to MSA's official journal, Microscopy and Microanalysis, and MSA's popular bi-monthly magazine, Microscopy Today.

- Peer Networking through the Society's Focused Interest Groups and Local Affiliated Societies.

- MSA Awards Programs, Scholarships, Speaker Opportunities, and much more!

Join MSA Today!

For more information: visit www.microscopy.org

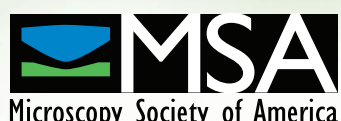

or call 1-800-538-3672 